\title{
ASPECTS REGARDING OPTIMAL DESIGN FOR THE WORM-GEAR DRIVE
}

\author{
Daniela Ghelase, Luiza Daschievici \\ Faculty of Engineering and Agronomy Braila, "Dunarea de Jos" University of Galati \\ 29 Calarasilor Street, Braila, Romania
}

Corresponding author: Daniela Ghelase, daniela.ghelase@ugal.ro

\begin{abstract}
It is known that, from the point of view of the accuracy of a machine-tool, at its design, the dynamic behaviour of each element of the kinematic chains prevails. Worm-gear drives are widely used in the different machine-tools and robots. Therefore, it is important that during meshing, as far as possible, there are no vibrations, shocks, power losses, noise and low durability. These requirements can be met if, for example, the gear ratio is constant during meshing, without transmission errors, which means that the worm-gear drive should have a high accuracy. The accuracy improvement of the worm-gear drive has long been a focus of attention for machine-tools designers. Thus, this paper presents various approaches to solving such problems, based on modelling and simulation, such as: estimating the load share of worm-gear drives and to calculate the instantaneous tooth meshing stiffness and loaded transmission errors; the desired worm-gear drive design configuration by altering the optimum set of wormgear drive design parameters which are suitable for the required performance by associating it with SVM (Support Vector Machine); optimization approach for design of worm-gear drive based on Genetic Algorithm; design optimization of worm-gear drive with reduced power loss; etc. The optimization of the worm-gear design is an important problem for the research because the design variables are correlated to each other. An optimal design algorithm developed by the authors of this paper, for worm-gear drive, is also presented.
\end{abstract}

Key Words: worm-gear drive, design, optimization, accuracy, machie-tools, robots.

\section{INTRODUCTION}

Worm-gear drives are widely used in the different machine-tools and robots because they have several advantages in comparison to other gear types. But, due to manufacturing errors and various factors such as elastic deformation under load, tooth profile modification, misalignment and wear, the kinematic accuracy of the worm-gear drive decreases and transmission errors occur. Therefore, during the machine-tool working, vibrations, shocks, power losses, noise, low durability may occur. In addition, the accuracy of the machine-tool is affected. The improvement of worm-gear drives has long been a focus of attention for machine and equipment designers and researchers, acting in the several directions of research and optimization, such as: load capacity, power loss, materials and lubrication, tooth geometry and contact, gearing tooth stiffness, manufacturing technologies.

Hence, Bharati M., et.al, [1] developed a SVM model which is able to offer accurate prediction of the performance parameters for a given set of design values. Yang F., et.al, [2] developed loaded tooth contact analysis (LTCA) for worm-gear drives. The authors determined load sharing between the teeth, real contact ratio and stresses for better worm-gear design. An evolutionary technique like Genetic Algorithm (GA) is also used for optimizing the worm and worm wheel with multi-objectives by Mogal Y.K. and Wakchaure V.D. in [4]. To reduced power loss, Rai P. and Barman A.G. [5] presented a design optimization algorithm for worm-gear drive, based on Simulated Annealing (SA).

\section{DESIGN OF WORM-GEAR DRIVE}

A variety of design models for the worm-gear drive can be developed by analytical approach. The various design parameters affect the analytical analysis of the worm-gear drive, such as: gear ratio, power, speed, module, etc. Several worm-gear design models can be developed, assigning a range of values for each parameter considered, to evaluate the design performance indicators for worm-gear drive. A database with design models can be created to run the SVM (Support Vector Machine) prediction model using the program based on analytical analysis. Unlike with ANN (Artificial Neural Network) where the architecture has to be developed in advance, the SVM model is easy to build and at the same time it generates a unique solution after training. The goal of SVM is to find out a function that gives a deviation of error from the actual given output and at the same time is as flat as possible. This is achieved by 
mapping the training patterns from the input space to a high dimensional feature space in such a way that the data which could not be separated by a linear function in the input space can be separated in the feature space [1]. Figure 1 shows presented a prediction model executed in MATLAB. As may be seen, input parameters for the SVM model are gear ratio, module, power and speed. The output parameters are: bending fatigue, wear capacity, heat generation. A total of 200 data points were obtained from analytical analysis of which 190 were used to develop the model.

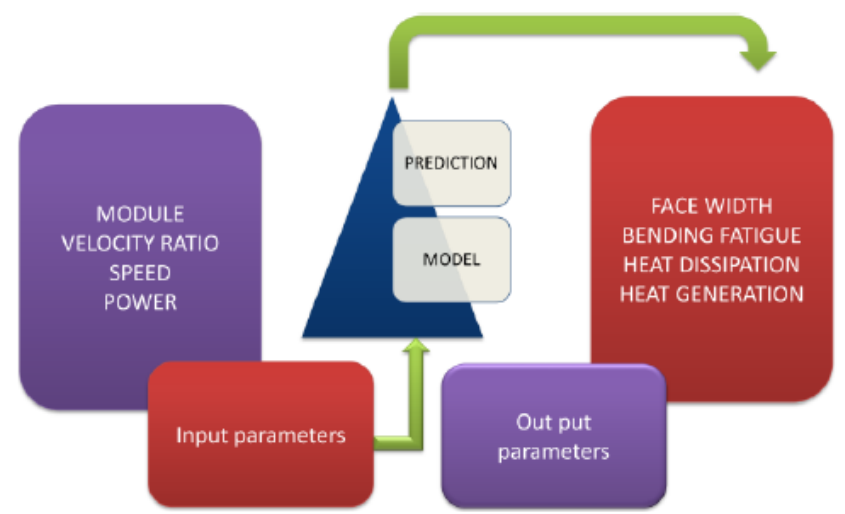

Fig. 1. SVM forward mapping prediction model [1]

The design of worm-gear drives with compact size and high loading capacity is developed in [2]. As it is known, the worm-gear drives are sensitive to manufacturing and assembly errors owing to the fact that they are designed on the assumption that the conjugated worm-gearing tooth surfaces are in line contact [3] (Figure 2).

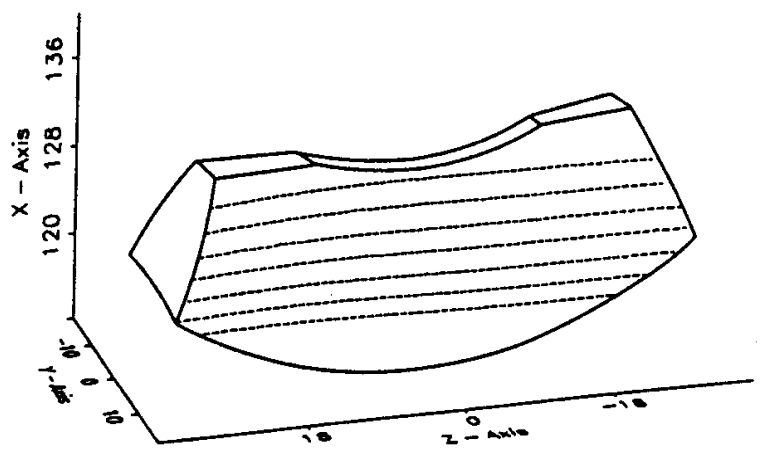

Fig. 2. Theoretical linear contact of worm-gearing tooth [3]

In reality, line contact is almost impossible to achieve on account of manufacturing and assembly errors and deflection under loading; instead, worse situations such as edge contact occur which severely reduce the loading capacity and, hence, the service life of wormgear drives. The contact lines degenerate into contact areas whose size and position vary with load [2].

In order to achieve localized tooth contact for involute worm-gear drives, this approach applies loaded tooth contact analysis (LTCA) and provides more accurate load capacity rating of worm-gear drives. LTCA [2] is based on the application of finite element analysis to determine load sharing between the teeth, real contact ratio and stresses.

For design of worm-gear drive with localized tooth contact, the following basic ideas are adopted for modifying the worm gearing tooth profiles [2]:

- reduce the worm diameter and increase the lead angle of the worm;

- make the modified worm have the same normal pitch at the reference point as the original one;

- $\quad$ change the hob mounting angle, $\theta_{\mathrm{h}}$, to modify the wheel tooth surface.

The design optimization of the worm-gear drive is an important problem for the researchers because the design variables are correlated to each other.

\section{OPTIMIZATION APPROACHES FOR DESIGN OF WORM-GEAR DRIVE}

Optimization is defined as process, which finds value of variables that minimize or maximize the objective function while satisfying constraints. Conventional optimization methods have been widely used in various mechanical design problems. They are deterministic in nature and use only a few geometric design variables due to their complexity and convergence problems. When the number of design parameters increases, the complexity increases drastically [4]. It is necessary to use more efficient methods to solve such problems.

Genetic Algorithm (GA) is one of evolutionary techniques. Genetic Algorithm is based on evolutionary processes and Darwin's concept of natural selection. It works on the principle that, only the fittest populations will survive while the bad populations are weeded out. The same concept is extended to the mathematical optimization problems where only good design points are selected while the bad design points are neglected. An initial population is chosen randomly at the beginning and fitness of initial population individuals is evaluated. Then an iterative process starts until the termination criteria have been run across. After the evaluation of individual fitness in the population, the genetic operators, selection, crossover and mutation are applied to breed a new generation. The newly created individuals replace the existing generation and reevaluation is started for fitness of new individuals. The loop is repeated until acceptable solution is found $[1,4]$.

SVM and Genetic Algorithms (GA) are used to build a forward-reverse mapping model for optimization of gear design performance [1]. As shown in Figure 3, the gear design parameters are sent to the softwarebased forward mapping model that has been built to 
map the relationship between the design parameters and performance metrics. For the given operating conditions, the forward mapping model predicts the performance. The desired optimum performance is defined so that the designer always operates under these conditions. This is achieved by linking the artificial intelligence based reverse mapping model with the forward mapping model. The reverse mapping model estimates the optimum performance metrics from a range of design parameter solutions that satisfy the require model.

Another optimization technique is Simulated Annealing. It is a stochastic optimization technique which can solve both constrained and unconstrained optimization problems [5]. This technique is based on the principle of heating of a material and then letting it cool down, thereby minimizing the defects and thus further reducing the system energy. There is random generation of new point at every iteration in simulated annealing. The distance between new and current point can be measured with the help of probability distribution with a scale. This probability distribution is proportional to the temperature. When the value of objective function is lowered, the algorithm accepts candidate point for solution along with those points which raise the value with a certain probability. Thus, the algorithm will not be trapped in local minima and produces a global optimum solution.

There are many literatures available related to design optimization of various gear drives using conventional and non-conventional optimization techniques.

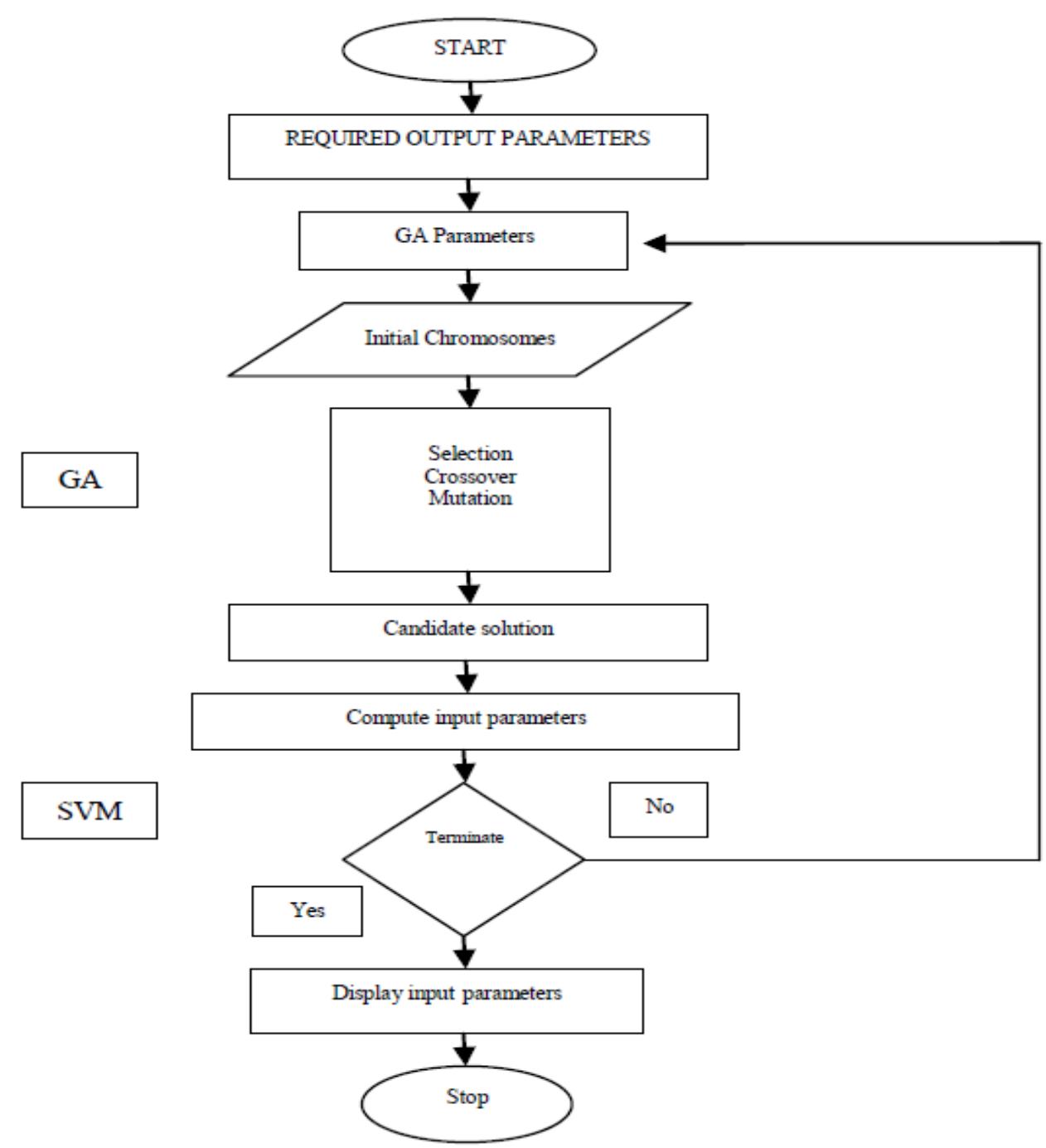

Fig. 3. Flow chart of the SVM and GA reverse mapping methodology [1]

\section{MULTI-OBJECTIVE OPTIMIZATION FOR DESIGN OF WORM-GEAR DRIVE}

In the paper [4] it is proposed an optimal design of worm-gear drive with multiple objectives, such as:
- To minimize the volume of worm and worm wheel;

- To minimize the centre distance between worm and worm wheel;

- To minimize the deflection of worm. 
The optimal design methodology is carried out by the authors using Genetic Algorithm, a technique little applied in the case of the worm-gear drive. In Table 1 there are the specifications of worm-gear drive. The mine steps of the mathematical modeling are the following:

A. Design Variables

The design variables are gear ratio (i), face width (b), pitch circle diameters of worm $\left(\mathrm{d}_{\mathrm{w}}\right)$ and worm wheel $\left(d_{g}\right)$ :

$$
F(x)=F(i, b, d w, d g)=F\left(x_{1}, x_{2}, x_{3}, x_{4}\right)
$$

Upper and lower bounds of design variables are $40 \leq$ $\mathrm{i} \leq 70,100 \leq \mathrm{b} \leq 200,150 \leq \mathrm{d}_{\mathrm{w}} \leq 280$ and $1000 \leq \mathrm{d}_{\mathrm{g}} \leq$ 1500 .

Table 1. Specifications of worm-gear drive [4]

\begin{tabular}{|l|l|c|c|}
\hline $\begin{array}{l}\text { Sr. } \\
\text { No. }\end{array}$ & Parameters & Worm & $\begin{array}{c}\text { Worm } \\
\text { wheel }\end{array}$ \\
\hline 1 & $\begin{array}{l}\text { Pitch circle diameter } \\
\text { (mm) }\end{array}$ & 240 & 1560.19 \\
\hline 2 & Number of starts/teeth & 1 & 65 \\
\hline 3 & Centre distance (mm) & \multicolumn{2}{|c|}{901} \\
\hline 4 & Face width (mm) & 75.408 & - \\
\hline 5 & Linear pitch (mm) & 48 & 0.7385 \\
\hline 6 & Speed (rpm) & 5.71 & - \\
\hline 7 & Lead angle (0) & \multicolumn{2}{|c|}{160} \\
\hline
\end{tabular}

\section{B. Objective Function}

The main objective function is to minimize volume of worm and worm wheel.

\section{Constraints}

Constraints are the conditions that must be met in the optimum design and include restrictions on design variables. The remaining objectives are taken as constraints such as centre distance, deflection of worm and beam strength of worm wheel.

After the implementation of the genetic algorithm, the optimized values of the design variables were obtained, as can be seen in Figure 4, where 1 is gear ratio, 2- face width, 3- pitch circle diameters of worm, 4- pitch circle diameters of wheel.

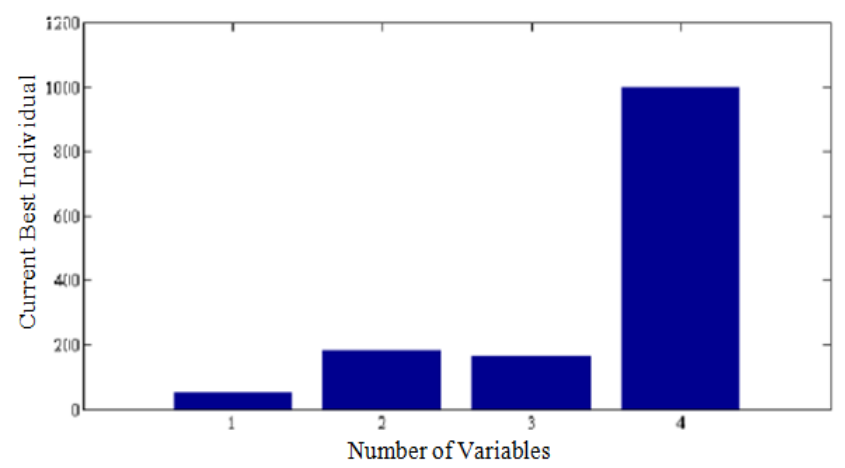

Fig. 4. Current best individual vs. number of variables [4]
Comparing the current values of the design variables with those obtained by using Genetic Algorithm, it can be seen that, for the numerical case considered in [4], there is a reduction of $17.91 \%$ in the volume of the worm and worm wheel, $20.34 \%$ reduction in the central distance between worm and worm wheel and $51.05 \%$ reduction of worm deviation. Therefore GA is one of the best tool for multi-objective optimization problem and can be used for optimal design of the worm-gear drive.

Taking different objectives, design variables and constraints other than above stated more reliable gear pair design may be obtained.

In [1] GA was again used by the authors for multiobjective optimization of worm-gear drive design. In addition, a model SVM has been developed that can provide an accurate prediction of performance parameters for a given set of design variables. The main object is to attain high wear capacity by considering module, power, gear ratio, and speed as design variables (Figure 1). Center distance and strength of worm-gear drive are the constraints taken into consideration.

As shown in [1], the predicted values from the SVM model were found to be very close to the actual values obtained through analytical analysis. As comparison between theoretical and SVM approach the correction values are dominant while error values are less in number. Using a SVM model, it is possible to estimate quickly the performance of the worm and worm wheel design. The benefit of such a prediction model is significant reduction of processing time as well as enhanced flexibility in the design performance.

\section{DESIGN OPTIMIZATION OF WORM-GEAR DRIVE WITH HIGH STIFFNESS}

Based on the algorithm for the evaluation of the worm gearing tooth stiffness, presented in [6], the elasticity characteristic of worm-gearing tooth was drawn (Figure 5).

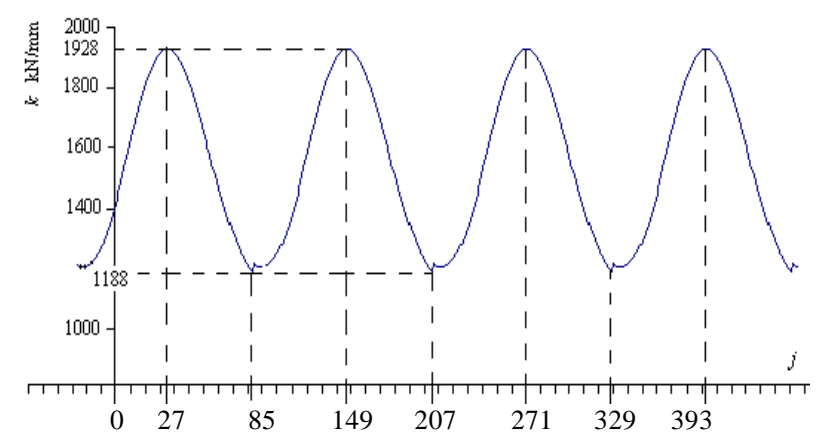

Fig. 5. Elasticity characteristic of the worm-gearing tooth [6]

The elasticity characteristic represents the variation of the worm-gearing tooth stiffness depending on the 
rolling angle $(\mathrm{j} \cdot \Delta \varphi)$, where “ $\mathrm{j}$ " is the rolling angular parameter. It is a cvasisinusoidal curve with the high jumps when a pair of teeth is getting into or out of the engagement process. The investigation of the elasticity characteristic is very important for the study of an elastic system, such as: gearing, kinematic chain, machine-tool. Hence, the introduction of this concept contributes to the completion of the used gearing study and it leads to increase of the gearing tooth stiffness.

Two optimization algorithms of geometrical parameters for the worm-gearing tooth with circular arch profile (general case) are presented in [7] :

- medium stiffness algorithm, for the highest possible stiffness;

- stiffness variation algorithm, for a constant stiffness.

Figure 6 shows the axial section of the worm with constant pitch, having a circular arch profile with the centre $\mathrm{O}_{1}$ for the right flank and $\mathrm{O}_{2}$ for the left flank, where:

$$
\begin{aligned}
& u=1.25 \cdot m / \cos \alpha ; \\
& p=m / 2 \\
& b=\pi \cdot m / 4-1.25 \cdot m \cdot \operatorname{tg} \alpha \\
& R=\sqrt{a^{2}+u^{2}}
\end{aligned}
$$

$p$ is the helical parameter of the motion that generates the helical surface of the worm-gearing tooth; $m$ - worm-gearing tooth module.

The proposed algorithms are based on research findings achieved by the authors of the paper [8].

Generally, the methodology regarding the achievement of stiffness as high as possible is the following (Figure 7):

- selection of a high value for diametral quotient $q$;

- increasing radius of profile curvature $R$;

- adoption of low value for worm profile angle $\alpha$;

- reduction of gear tooth number $z_{2}$.

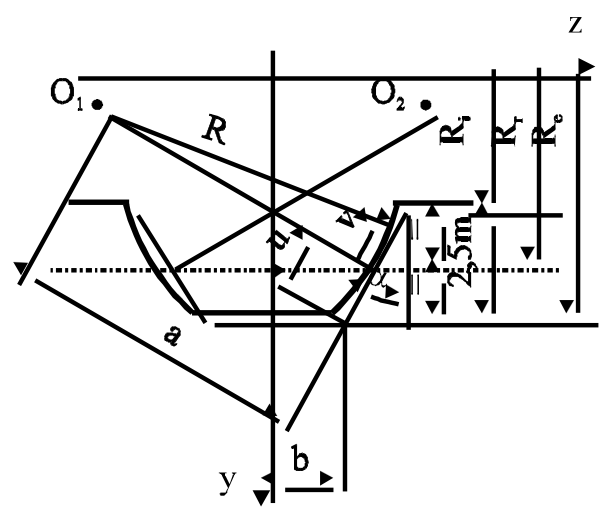

Fig. 6. Worm flank geometry [8]

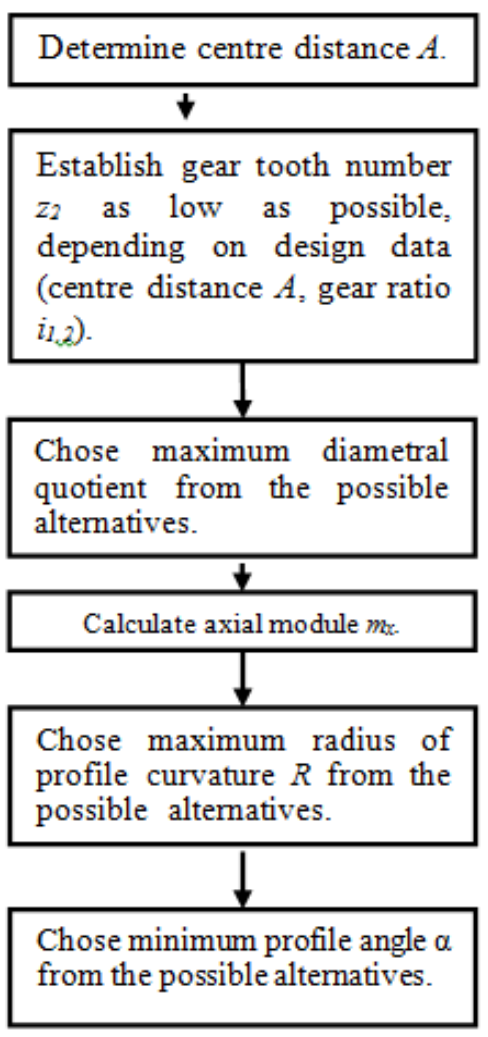

Fig. 7. Optimal design using medium stiffness algorithm [8]

From the viewpoint of the influences on the increase of medium stiffness, the hierarchy of the geometrical parameters is the following:

1) diametral quotient $q$;

2) radius of profile curvature $R$;

3) gear tooth number $z_{2}$;

4) worm profile angle $\alpha$.

In order to improve the performance of the wormgear drive, regarding the gearing tooth stiffness, in the paper [2], an approach based on LTCA (localized tooth contact area) is presented. The results of this study can be taken into account in the optimal design of worm-gear drives. With LTCA, the deformation of teeth, stresses in gears and load distribution between teeth can be estimated more accurately. After Finite Element modelling for involute worm gearing tooth and determination of the localized tooth contact area, it can be calculated the mesh stiffness of engaging tooth pairs. To simplify this calculus, the following assumptions are introduced:

- Tooth deformation is elastic;

- The centre of the contact ellipses will not shift after the elastic deformation.

Figure 8 shows the gearing tooth deformation under the load. If in [6] the study of the gearing tooth stiffness has been developed on a deformable worm gearing without manufacturing errors, in [2] the meshing stiffness for a worm-gear drive is actually a function of contact position which is defined as the meshing stiffness function along the contact path 
(MSFACP). Figure 9 shows the meshing stiffness of an involute worm-gear drive.
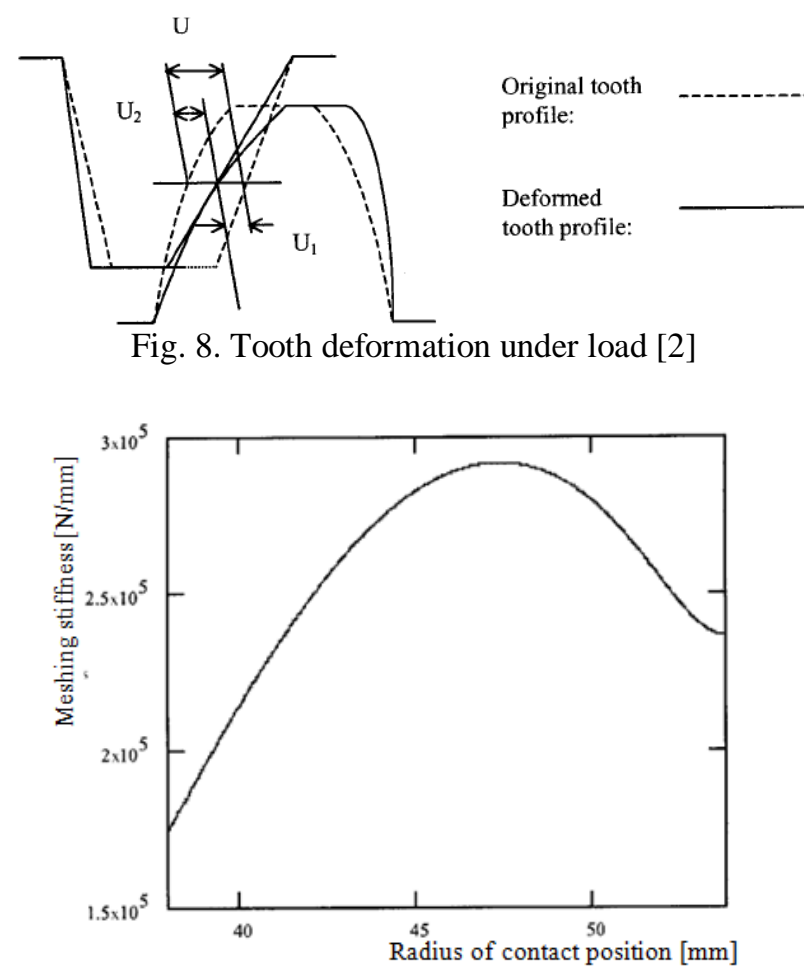

Fig. 9. Meshing stiffness of an involute worm-gear drive [2]

It is known that two factors may significantly affect the gear transmission. One is the tooth profile error caused by manufacturing and assembly errors and the other is the tooth elastic deformation for both the worm and the worm gear, determined by the load acting on the contacting teeth. Based on the calculation of the stiffness of worm gearing tooth, the load share of the worm gear drives can be estimated [2]. The real transmission error under load is determined by the actual total displacement of the contact tooth pairs, which is affected by the elastic deflection of the loaded tooth body. The total displacement can be considered as the sum of the elastic deformation and the theoretical transmission error (the tooth profile separation caused by manufacturing error or misalignment).

\section{DESIGN OPTIMIZATION OF WORM GEAR DRIVE WITH REDUCED POWER LOSS}

To reduced power loss, a design optimization algorithm for worm-gear drive was developed in [5]. Simulated Annealing (SA) has been used to minimize the power loss of worm-gear drive. Owing to the relative sliding motion between the gear teeth, the friction is high in worm-gear drive leading to high power loss. Therefore, the efficiency of worm-gear drive is reduced. Objective function is to minimize loss of power in worm-gear drive. Design variables are gear tooth number $\left(\mathrm{z}_{2}\right)$, helix angle $\left(\Phi_{\mathrm{n}}\right)$ and coefficient of friction $(\mu)$.

Upper and lower bounds of design variables are $21 \leq$ $\mathrm{z}_{2} \leq 80,15 \leq \Phi_{\mathrm{n}} \leq 25,0.03 \leq \mu \leq 0.05$.

Bending strength, contact pressure and linear deflection of worm are taken as the design constraints.

The authors of that research consider that the optimal worm-gear drive model obtained is found to be better in comparison to earlier used optimization techniques, due to the following reasons:

1) It can be seen that the value of friction coefficient is lower in comparison to other optimization technique used earlier (Differential Evolution, Particle Swarm Optimization and GA);

2) Helix angle obtained using $S A$ is greater in comparison with DE, PSO and GA;

3) The percentage reduction of power loss using SA ranges from $78.02 \%$ to $22.98 \%$.

Therefore, the optimized worm-gear drive will have better service life and the attained results show that the performance of SA is better in comparison with other approaches used earlier for power loss minimization of worm-gear drives.

\section{CONCLUSIONS}

There are many literatures available related to design optimization of worm-gear drives using conventional and non-conventional optimization techniques. This paper presents several optimizing methodologies of worm-gear drive design in order to improve its performance.

Multi-objective optimization for design of worm-gear drive- if an optimization is desired targeting several objectives, such as geometric parameters of the gearing. The basis of this approach is Genetic Algorithm, one of the best tool for multi-objective optimization.

To provide an accurate prediction of performance parameters for a given set of design variables, a design optimization technique based on SVM (Support Vector Machine) with GA can be used.

In order to improve the performance of the wormgear drive, regarding the gearing stiffness, the medium stiffness algorithm can be applied.

Finite Element modelling for involute worm gearing tooth with LTCA (localized tooth contact area), the deformation of teeth, stresses in gears and load distribution between teeth can be estimated more accurately.

Based on the calculation of the stiffness of worm gearing tooth, the load share of the worm-gear drives can be estimated.

The real transmission error under load is determined by the actual total displacement of the contact tooth pairs, which is affected by the elastic deflection of the loaded tooth body. 
To improve the efficiency of worm-gear drive, the design optimization with reduced power loss, based on Simulated Annealing (SA), is indicated for using.

\section{REFERENCES}

1. Bharati M, Deepika Poornima V and Jyothrimai S, (2015), A Performance Prediction of Worm-Worm Wheel and its Multi Objective Design Parameter Optimization Based on Genetic Algorithm, nternational, Journal of Scientific and Research Publications, 5(2), 0215.

2. Yang F, Su D and Gentle C R., (2001), Finite element modelling and load share analysis for involute worm gears with localized tooth contact, Proceedings of the Institution of Mechanical Engineers, Part C: Journal of Mechanical Engineering Science, 215(7), doi.org/10.1243/0954406011524162.

3. Seol I H., (1996), Computerized design, generation and simulation of meshing and contact of worm-gear drives with improved geometry, Computer Methods in Applied Mechanics and Engineering, 138(1-4), 73-103.

4. Mogal Y K and Wakchaure V D., (2013), A multi-objective optimization approach for design of worm and worm wheel based on genetic algorithm, Int. J. Man Mach. Interface, 3(1), pp. 8-12.

5. Rai P and Barman A G., (2019), Design Optimization of Worm Gear Drive with Reduced Power Loss, Proc. Int. Conf. $10^{\text {th }}$ on Mechatronics and Manufacturing Series: Materials Science 635and Engineering (Bangkok, Thailand, 21-23 January 2019) (Bristol: IOP Publishing) p 1.

6. Ghelase D Daschievici L., (2020), Aspects regarding the evaluation of the gearing tooth stiffness, New Advances in Mechanisms, Mechanical Transmissions and Robotics: Proc. Int. Conf. $13^{\text {th }}$ on Mechanism and Mechanical Transmission and Int. Conf. $24^{\text {th }}$ on Robotics (Timisoara, Romania, 14-16 October 2020) (Mechanisms and Machine Science, 88, ed E C Lovasz et al (Cham: Springer), pp 313323.

7. Ghelase D Daschievici L and Ghelase I., (2010), Modelling and assisted design of the worm-gearing, Proc. Int. Conf. $9^{\text {th }}$ on System Science and Simulation in Engineering (Iwate, Japan, 4-6 October2010), ed H Fujita and J Sasaki (Iwate: WSEAS Press) pp 160165.

8. Ghelase D Daschievici L and Diaconescu I., (2006), Optimal Design of the Worm Gearing with Circular Profile Using Medium Rigidity Criterion Proc. Int. Conf. $15^{\text {th }}$ on Manufacturing Systems (Bucharest, Romania, 26-27 October2006), ed C Ispas et al (Bucharest: Editura Academiei Romane) pp 453-456.
Received: March 22, 2021 / Accepted: December 20, 2021 / Paper available online: December 25, 2021 (c) International Journal of Modern Manufacturing Technologies 